\title{
Apoptosis is an innate defense function of macrophages against Mycobacterium tuberculosis
}

\author{
SM Behar ${ }^{1}$, CJ Martin ${ }^{1}$, MG Booty ${ }^{1}$, T Nishimura ${ }^{1}$, X Zhao $^{1}$, H Gan $^{1}$, M Divangahi $^{1,2}$ and HG Remold $^{1}$
}

Two different forms of death are commonly observed when Mycobacterium tuberculosis (Mtb)-infected macrophages die: (i) necrosis, a death modality defined by cell lysis and (ii) apoptosis, a form of death that maintains an intact plasma membrane. Necrosis is a mechanism used by bacteria to exit the macrophage, evade host defenses, and spread. In contrast, apoptosis of infected macrophages is associated with diminished pathogen viability. Apoptosis occurs when tumor necrosis factor activates the extrinsic death domain pathway, leading to caspase-8 activation. In addition, mitochondrial outer membrane permeabilization leading to activation of the intrinsic apoptotic pathway is required. Both pathways lead to caspase-3 activation, which results in apoptosis. We have recently demonstrated that during mycobacterial infection, cell death is regulated by the eicosanoids, prostaglandin $E_{2}$ (proapoptotic) and lipoxin (LX)A 4 (pronecrotic). Although $\mathrm{PGE}_{2}$ protects against necrosis, virulent $M$ tb induces $\mathrm{LXA}_{4}$ and inhibits $\mathrm{PGE}_{2}$ production. Under such conditions, mitochondrial inner membrane damage leads to macrophage necrosis. Thus, virulent $M t b$ subverts eicosanoid regulation of cell death to foil innate defense mechanisms of the macrophage.

\section{APOPTOSIS IS AN IMPORTANT HOST DEFENSE MECHANISM}

Initially, inhalation of infectious aerosol delivers Mycobacterium tuberculosis $(M t b)$ into the distal airways of the lung, where it infects alveolar macrophages. The macrophage engulfs the bacilli using phagocytic receptors, of which the complement and mannose receptors are the most important. ${ }^{1,2}$ Virulent $M t b$ survives in macrophages by preventing acidification of the phagosomal compartment and by impairing the fusion of the phagosome with lysosomes. ${ }^{3}$ Thus, $M t b$ adapts to the intracellular environment of the macrophage and creates a niche for itself.

The manner by which the macrophage responds following infection by $M t b$ makes a crucial contribution to the host immune response and the outcome of infection. Three major outcomes are observed following productive $M t b$ infection of human or murine macrophages in vitro: (i) necrosis, a form of death characterized by plasma membrane disruption; (ii) apoptosis, a form of death in which plasma membrane integrity is preserved; and (iii) survival of infected macrophages.
Characterization of these three different phenotypes is challenging because of the asynchronous nature of intracellular infection and proposed heterogeneity among bacteria and macrophages. Other factors such as the percentage of infected macrophages and variation in the number of bacteria internalized by each macrophage can also affect the kinetics of cell death when studied in vitro. Nevertheless, a spectrum of all three phenotypes can be observed following infection of macrophages with virulent $M t b$. Although highly virulent $M t b$ strains predominantly induce necrosis, ${ }^{4}$ apoptosis is also detected among macrophages infected with virulent $M t b$ (Figure 1). Whereas apoptosis enables control of bacterial replication, virulent $M t b$ strains inhibit the completion of apoptosis, a virulence mechanism used by the pathogen to foil host defenses, and results in necrosis and ongoing infection of the surrounding macrophages. ${ }^{4}$

The different cellular fates of $M t b$-infected macrophages are of great interest as the death modality influences the outcome of infection. In particular, apoptotic death reduces the viability of different mycobacterial species, ${ }^{5,6}$ including $M t b{ }^{7,8}$

${ }^{1}$ Division of Rheumatology, Immunology, and Allergy, Department of Medicine, Brigham and Women's Hospital, Harvard Medical School, Boston, MA, USA. Present address: Meakins-Christie Laboratories, Department of Medicine, McGill University, Montreal, Quebec, Canada. Correspondence: HG Remold (hremold@rics.bwh. harvard.edu)

Received 30 November 2010; accepted 3 January 2011; published online 9 February 2011. doi:10.1038/mi.2011.3 

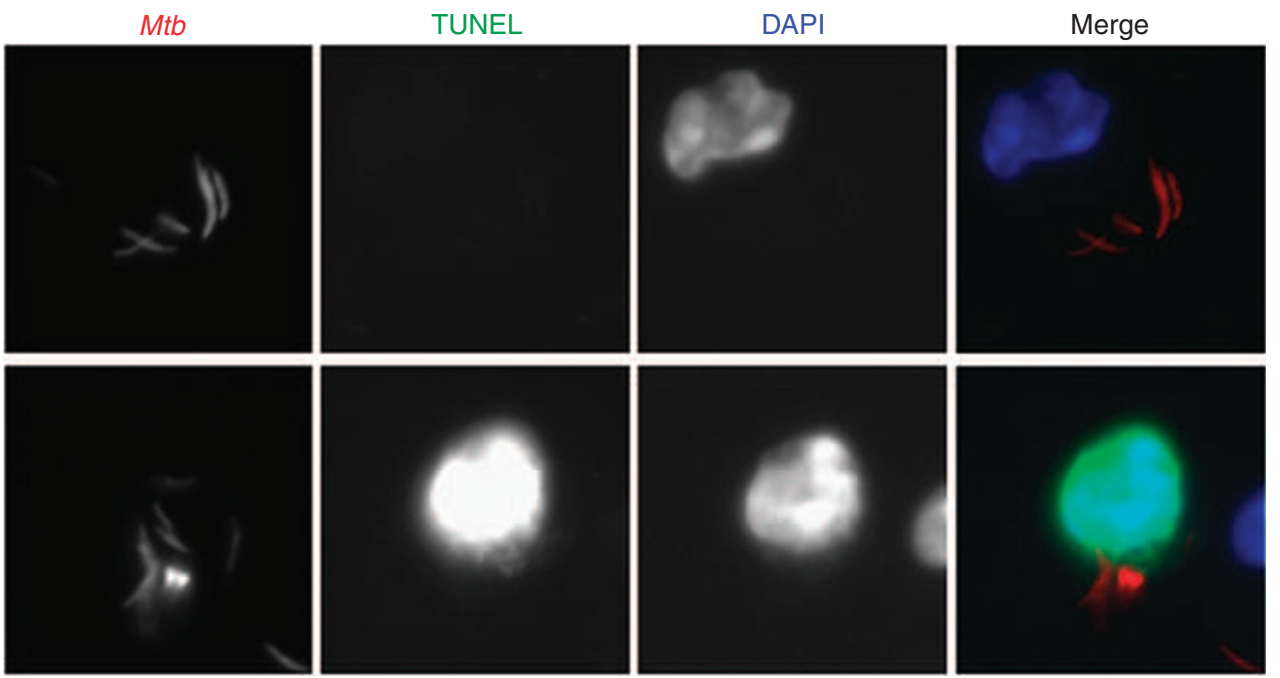

Figure 1 Apoptotic cell death following Mtb infection. CD11 b thioglycolate-elicited peritoneal mouse macrophages were infected at an MOI of 10:1 with mCherry-expressing H37Rv. Cells were fixed, permeabilized, and TUNEL stained according to the In situ Cell Death Detection Kit (Roche, Mannheim, Germany) 2 days later. Coverslips were mounted on slides using ProLong Gold with DAPI (Invitrogen, Carlsbad, CA) to label nuclei. Macrophages containing intracellular bacteria were identified by wide-field microscopy using a Nikon Microscope (Nikon, Tokyo, Japan) at $\times 40$ magnification and then the nuclear staining assessed. Both TUNEL-negative (top row) and TUNEL-positive (bottom row) infected cells are found. DAPI, 4',6-diamidino-2-phenylindole; MOI, multiplicity of infection; Mtb, Mycobacterium tuberculosis; TUNEL, terminal deoxynucleotidyl transferase dUTP nick-end labeling.

In addition, CD95L binding to CD95 expressed by Mtb-infected macrophages induces apoptosis and is accompanied by a substantial reduction in bacterial viability. ${ }^{9}$ This suggests that T cells could target infected macrophages for apoptosis, which in addition to the elaboration of cytokines, may be an important mechanism for restricting $M t b$ infection. The discovery that many attenuated strains of Mycobacteria induce more apoptosis than their virulent counterparts has led to the idea that virulent Mycobacteria inhibit either the induction or the completion of macrophage apoptosis. Indeed, there exists a reciprocal relationship between virulence and apoptosis. As such, $M t b$ infection predominantly results in necrosis, whereas attenuated mutants including BCG (Bacille Calmette-Guerin) and H37Ra primarily induce apoptosis. At present, investigators are identifying single gene mutations in $M t b$ that shift the balance from necrosis to apoptosis. ${ }^{10,11}$ Although it is not yet clear whether virulent $M t b$ blocks the induction of apoptosis or inhibits its downstream events, it can now be clearly argued that $M t b$ evades host defenses by inducing necrosis, a form of cell death that allows bacteria to exit from macrophages and disseminate.

Recent studies have evaluated whether differences in the fate of infected cells alter the outcome of infection using whole animal models. It is clear that proapoptotic mutants of $M t b$ prime a greater T-cell response and enhance host control of infection. ${ }^{10}$ This has generated considerable interest in whether proapoptotic mutants of $M t b$ could be used as a vaccine strategy. For example, vaccination with attenuated BCG or $M t b$ that induces greater macrophage apoptosis, or with apoptotic bodies purified from BCG-infected macrophages, ${ }^{12}$ may stimulate a quantitatively better T-cell response. There is also evidence that dendritic cells acquire antigens from infected apoptotic macrophages. Such dendritic cells can then prime naive $\mathrm{T}$ cells through a process known as cross-priming, which may stimulate different $\mathrm{T}$-cell subsets, such as $\mathrm{CD}^{+} \mathrm{T}$ cells, more efficiently. ${ }^{7,10,12}$ Just as bacterial factors can affect death modality, host factors also affect whether a cell undergoes apoptosis or necrosis, even following infection with virulent $M t b$. In particular, eicosanoids seem to be critical regulators of apoptosis following $M t b$ infection..$^{7,8,13}$ These studies have provided important evidence that during pulmonary $M t b$ infection, apoptosis of infected macrophages leads to innate control of early bacterial growth and acts as a reservoir of antigen that facilitates initiation of acquired T-cell immunity through cross-priming by dendritic cells.

Although cell death is a tightly regulated process, the hostpathogen interaction adds several layers of complexity. The manner by which the death modality of infected cells affects the outcome of infection, particularly during different clinical states in individuals (e.g., latent vs. active tuberculosis), remains a pertinent question. In this study, other investigators working on the genetics of susceptibility to $M t b$ provide an important perspective. Gene expression profiling finds that several genes related to apoptosis are less expressed in active tuberculosis patients than in latently infected individuals, suggesting that decreased apoptotic activity is associated with the reactivation of latent infection. ${ }^{14}$ Using a more targeted approach, Abebe et al. found that patients with active tuberculosis in Ethiopia had elevated expression of genes associated with the extrinsic apoptosis pathway, including tumor necrosis factor (TNF), Fas, FasL, and caspase-8. However, expression of FLIP, an intrinsic inhibitor of caspase-8, was also significantly elevated. ${ }^{15}$ Although the upregulation of TNF, Fas/FasL, and caspase- 8 may be the signature of an immune response capable of inducing apoptosis in infected cells, the authors propose a model in 
which $M t b$ inhibits the extrinsic apoptosis pathway by upregulating FLIP to evade an apoptotic death. Finally, the eicosanoid biosynthetic pathways, which regulate the death modality of infected human and murine macrophages, have now been identified as important genetic loci that regulate susceptibility to tuberculosis and leprosy in individuals. ${ }^{16,17}$ Although genetic and functional data require greater scrutiny and functional correlation, they independently provide scientific motivation to better understand how cell death is regulated in $M t b$-infected macrophages.

\section{WHAT CELLULAR EVENTS LEADTO MACROPHAGE APOPTOSIS?}

Apoptosis can be initiated by several mechanisms; however, it is defined on the basis of a number of morphological and molecular criteria. The hallmarks of apoptosis include the segmentation of DNA, ${ }^{18}$ exposure of phosphatidylserine on the outer leaflet of the plasma membrane, and finally, deposition of cellular components into membrane-bound blebs. ${ }^{19,20}$ During apoptosis, the dying cell produces "find-me" and "eat-me" signals that aid its rapid clearance by phagocytes through efferocytosis. ${ }^{21}$ Similarly, a detailed analysis of "necrosis" reveals it to be heterogeneous, and certain subtypes that have unique cellular and molecular mechanisms and triggers can be defined. For instance, pyroptosis or necroptosis can be considered forms of necrosis that are dependent on caspase- 1 and RIP-1 (receptor-interacting protein-1), respectively. ${ }^{22-24}$ Given the finality of death, it is not surprising that the molecular pathways leading to apoptotic or necrotic death are highly regulated.

As discussed above, apoptosis acts as a host defense mechanism of last resort. By sequestering pathogens within apoptotic cells, a reduction in bacterial viability is achieved. Furthermore, apoptotic macrophages provide an important source of bacterial antigens that can stimulate $M t b$-specific T-cell immunity. ${ }^{12}$ However, there are accumulating data that suggest that virulent $M t b$ produces bacterial products that inhibit apoptosis and instead lead to macrophage necrosis. The presumptive subversion of the host-regulatory pathways by $M t b$ leads to evasion of innate macrophage defense mechanisms and to a delay in adaptive immunity. Therefore, determining the signaling pathways that regulate apoptosis during $M t b$ infection is critical for understanding how the pathogen evades host immunity.

\section{Induction of apoptosis through the extrinsic pathway in Mtb-infected macrophages}

The induction of apoptosis by attenuated $M t b$ in human monocyte-derived macrophages, which is mediated by the executioner caspases- 3 and -7 , requires two distinct signals: a cytokine-dependent signal and a lipid-dependent signal. ${ }^{25,26}$ The cytokine-dependent signal, which requires protein synthesis, is represented by TNF. Indeed, the induction of apoptosis in macrophages by $M t b$ requires the action of TNF to activate the extrinsic death receptor-dependent pathway (Figure 2). Although the mycobacterial strains H37Rv and H37Ra elicit comparable amounts of TNF, the avirulent strain $\mathrm{H} 37 \mathrm{Ra}$ is a much more potent inducer of apoptosis than the virulent $\mathrm{H} 37 \mathrm{Rv}^{27}$ One possible mechanism is that TNF receptor-2, which is shed by macrophages infected with virulent $M t b$, inactivates soluble TNF. ${ }^{28}$ The lipid-dependent (protein synthesis-independent) signal involves the action of group IV cytosolic phospholipase $\mathrm{A}_{2}-\gamma$, a cytosolic phospholipase $\mathrm{A}_{2}$ that releases arachidonic acid (the common substrate for eicosanoids, including prostanoids and lipoxins (LXs)) from the sn-2 position of glycerophospholipids of the plasma membrane. ${ }^{29}$

Recently, some of the early components of the extrinsic apoptotic pathway initiated by $M t b$ in the mouse macrophage cell line RAW264 have been identified. ${ }^{30}$ It was found that following infection with $M t b$, TNF production stimulates reactive oxygen species (ROS)-dependent activation of apoptosis signalregulating kinase (A002816), a member of the mitogenactivated protein kinase family causing FLIP ${ }_{\mathrm{s}}$ phosphorylation. Phosphorylated FLIP interacts with the E3 ubiquitin ligase c-CYBL facilitating proteasomal FLIP ${ }_{s}$ degradation involving the tyrosine kinase c-Abl. FLIP $_{s}$ degradation activates caspase-8, leading to caspase- $3 / 7$ activation and apoptosis. These findings illustrate which factors are involved in the $M t b$-dependent induction of apoptosis of the host macrophage, leading to promotion of caspase- 8 activation. However, in this study, the mechanisms downstream of caspase- 8 activation were not analyzed, nor was the manner by which apoptosis subverted by virulent $M t b$ to induce necrosis considered.

\section{The intrinsic pathway: the role of the mitochondria in the induction of apoptosis and necrosis}

Induction of apoptosis in vertebrate cells most commonly proceeds through the intrinsic apoptotic pathway, which is functionally defined by mitochondrial outer membrane permeabilization (MOMP). ${ }^{31}$ MOMP is a central event that can lead to apoptosis as it results in the release of cytochrome $c$, Smac-DIABLO, apoptosis inducing factor, and other factors from the mitochondrial intermembrane space and ultimately results in the activation of caspase-3/7 (Figure 2). Although these events can occur independently of any other changes in the mitochondria, they can also be associated with the opening of the mitochondrial inner membrane pore (permeability transition pore), which leads to mitochondrial permeability transition (MPT), loss of the mitochondrial intermembrane potential $\left(\Delta \psi_{\mathrm{m}}\right)$, and leads to necrosis. $M t b$ infection, whether virulent or avirulent, induces such changes in the mitochondrial membranes. As such, these changes are the key events that influence the death modality of infected macrophages. The different combinations of MOMP and MPT in model experimental systems, and their effect on cellular outcome are reviewed below. Changes in MOMP and MPT induced by virulent and avirulent $M t b$ infection will then be discussed in the context of these scenarios. Table $\mathbf{1}$ and Figure 3 summarize the different combinations of MOMP and MPT that are likely to occur.

In scenario I, MPT causes the mitochondria to become leaky to water, which results in swelling, dysfunction, and eventually to necrosis. ${ }^{31}$ Irreversible MPT can lead to outer mitochondrial membrane damage, which manifests itself as MOMP, here 


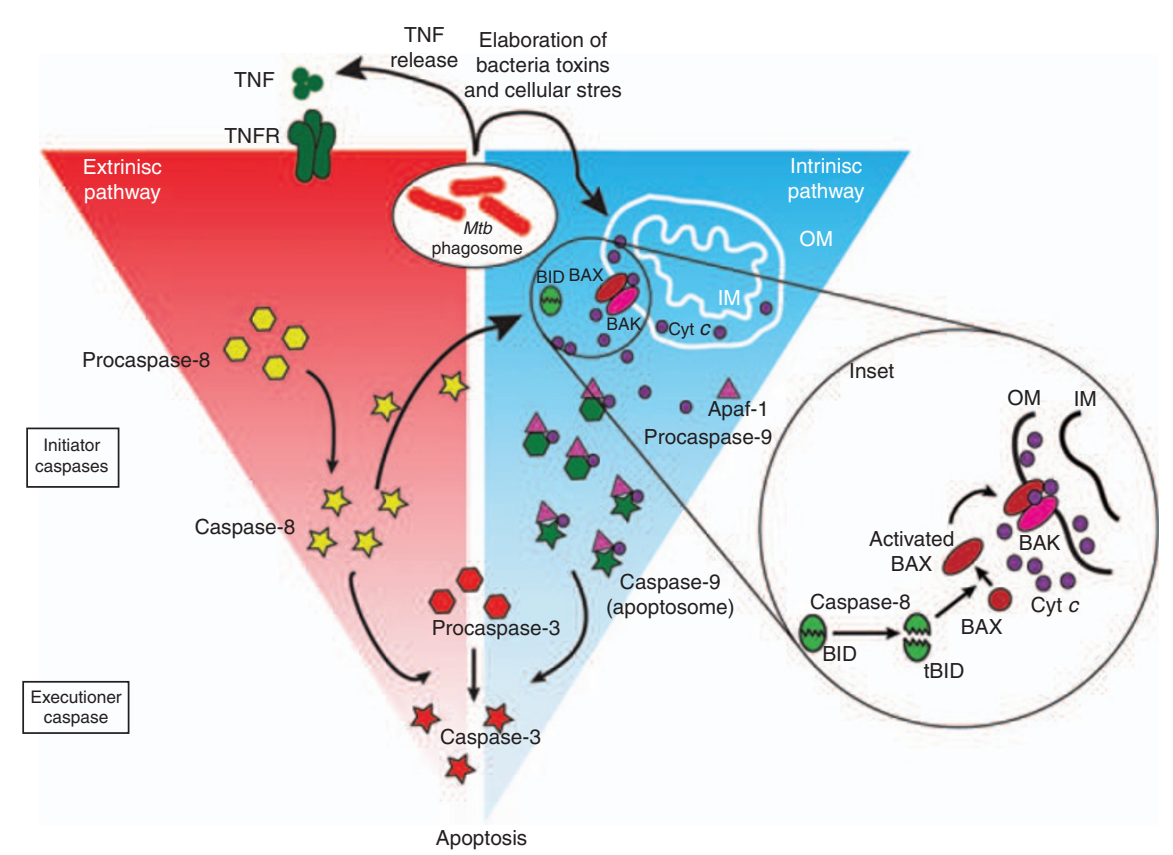

Figure 2 Extrinsic and intrinsic pathways of apoptosis. During Mtb infection, several signals generated by the infected macrophage can trigger apoptosis. For example, TNF production can activate the extrinsic pathway resulting in caspase-8 activation. What triggers the intrinsic pathway is unknown. Cellular stress or caspase-8 activation can result in BID cleavage, leading to BAX activation, and the ensuing events lead to cytochrome $c$ translocation from the mitochondrial intermembrane space into the cytosol where it activates caspase-9. Alternately, caspase-8 activation can directly lead to caspase-3 activation in certain cells (type I). cyt $c$, cytochrome $c$; IM, inner mitochondrial membrane; Mtb, Mycobacterium tuberculosis; OM, outer mitochondrial membrane; TNF, tumor necrosis factor; TNFR, TNF receptor.

Table 1 Different interactions between MOMP and MPT alter the cell death modality

\begin{tabular}{|c|c|c|c|c|}
\hline \multirow[t]{2}{*}{ Scenario } & I & II & III & IV \\
\hline & $\begin{array}{l}\text { MOMP dependent on } \\
\text { MPT }\end{array}$ & MOMP independent of MPT & $\begin{array}{l}\text { MPT dependent on } \\
\text { MOMP }\end{array}$ & $\begin{array}{l}\text { MPT independent of } \\
\text { MOMP }\end{array}$ \\
\hline Example & $\begin{array}{l}\text { Induction of apoptosis } \\
\text { in cells undergoing MPT } \\
\text { due to oxidative stress }\end{array}$ & $\begin{array}{l}\text { Induction of cytochrome } c \text { release from } \\
\text { the mitochondria in staurosporine-treated } \\
\text { CEM cells does not affect MPT }{ }^{34}\end{array}$ & $\begin{array}{l}\text { Inner mitochondrial mem- } \\
\text { brane damage caused by } \\
\text { caspase- } 3^{35}\end{array}$ & $\begin{array}{l}\text { Granzyme A damage } \\
\text { of the inner mitochon- } \\
\text { drial membrane }\end{array}$ \\
\hline Outcome & Necrosis & Apoptosis & Necrosis & Necrosis \\
\hline
\end{tabular}

Abbreviations: MOMP, mitochondrial outer membrane permeabilization; MPT, mitochondrial permeability transition.

a by-product of MPT. This scenario emerges when hepatocytes under oxidative stress, or due to other toxic treatments, undergo both necrosis and apoptosis. ${ }^{32}$

However, as shown in scenario II, MOMP and apoptosis can occur independently of MPT. This is the case when MOMP is induced by members of the Bcl-2 family of apoptosis-inducing proteins, which do not affect the mitochondrial inner membrane. ${ }^{33}$ In particular, processing of the Bcl-2-only protein BID leads to activation of the proapoptotic Bcl-2 family proteins BAX and BAK, causing MOMP and translocation of proapoptotic factors including cytochrome $c$ into the cytosol, activation of caspase-9, and eventually that of caspase- 3 . This process neither induces nor requires MPT. ${ }^{34}$

As depicted in scenario III, effector molecules damage the mitochondrial inner membrane and gain access to the mitochondrial intermembrane space if the mitochondrial outer membrane is permeable. This can be the mechanism by which Ndufs 1 , a component of the electron transport chain, is damaged by caspase- $3 .^{35}$ Damage of Ndufs 1 disrupts the electron transport chain in the inner membrane, leading to ROS formation and necrosis. Caspase- 3 is believed to enter the mitochondrial intermembrane space through pores generated in the mitochondrial outer membrane, which allow proapoptotic factors including cytochrome $c$ to escape into the cytosol. ${ }^{34}$

Finally, MPT can also occur independently of MOMP (scenario IV). This is believed to be the manner by which granzyme A damages the components of the mitochondrial inner membrane. ${ }^{36} \mathrm{Hsp} 70$ and Hsp90 are candidate molecules that serve as cytosolic chaperones for granzyme A and allow the protease to enter the mitochondrial intermembrane space without damaging the mitochondrial outer membrane leading to cleavage of Ndufs 3 , the $30-\mathrm{kDa}$ subunit of mitochondrial complex $1 .{ }^{36}$ 


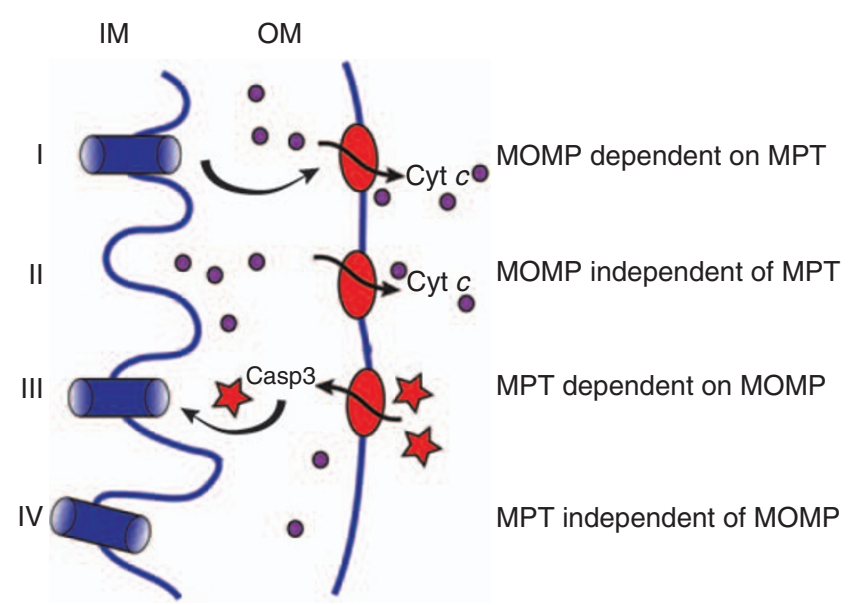

Figure 3 Mitochondrial membrane damage leads to cell death. Four different scenarios of inner or outer mitochondrial membrane damage lead to mitochondrion dysfunction (see Table 1). Opening of the permeability transition pore in the inner mitochondrial membrane (IM), leads to MPT, and can occur in isolation (IV) or can increase outer membrane (OM) permeability (MOMP), leading to leaking of cytochrome $c$ (I). In addition, MOMP leading to cytochrome $c$ release into the cytosol can occur independently of MPT (II). Finally, under certain conditions, OM permeability might allow entry of activated caspase- 3 into the mitochondrial intermembrane space, which can lead to damage of the IM and result in MPT. Casp3; caspase-3; cyt c, cytochrome $c$; IM, inner mitochondrial membrane; MOMP, mitochondrial outer membrane permeability; OM, outer mitochondrial membrane.

\section{Mitochondrial cellular death pathways in Mtb-infected macrophages}

In macrophages infected with attenuated $M t b$, apoptosis is associated with MOMP, yet MPT is not induced, as described in scenario II (Table 1). ${ }^{34}$ Inhibition of MOMP diminishes only apoptosis, but does not affect MPT. ${ }^{4}$ Silencing of the gene for the proapoptotic $\mathrm{Bcl}-2$ protein $\mathrm{BAX}$, which is required for the release of cytochrome $c$ and apoptosis inducing factor from the mitochondrial intermembrane space, abrogates $M t b$-induced apoptosis, but does not affect MPT or necrosis. ${ }^{4}$

In contrast, virulent $M t b$ induces both MOMP and MPT, leading to irreversible mitochondrial swelling and necrosis. ${ }^{4} \mathrm{MPT}$ can be inhibited by CsA (which binds to cyclophilin D in the mitochondrial inner membrane), has a requirement for mitochondrial $\mathrm{Ca}^{++}$loading, and is independent of Bcl-2 family member-induced apoptosis. ${ }^{37}$ Inhibition of MPT, downregulates only necrosis, but does not affect the degree of MOMP or apoptosis. ${ }^{38,39} \mathrm{It}$ is not clear at present whether in $M t b$-infected macrophages, MPT is dependent on the opening of pores in the mitochondrial outer membrane (MOMP_scenario III) or whether toxic molecular species enter the mitochondrial intermembrane space through chaperones (see the study by Martinvalet et al. ${ }^{36}$ —scenario IV). The different mechanisms induced by virulent and avirulent $M t b$ indicate that in $M t b$-infected macrophages, MOMP and MPT are independent phenomena; virulent $M t b$ is unique in its induction of MPT that leads to the destruction of the mitochondrial outer membrane causing secondary cytochrome $c$ release (see scenario I, also Figure 3). ${ }^{4}$
In summary, induction of apoptosis or necrosis in $M t b$-infected macrophages depends on highly specific mechanisms leading to different types of mitochondrial membrane perturbation. Attenuated and virulent $M t b$ alike cause transient MOMP characterized by cytochrome $c$ release from the mitochondrial intermembrane space, which requires BAX. In contrast, only virulent $\mathrm{H} 37 \mathrm{Rv}$ causes MPT.

\section{The apoptotic envelope of the macrophage: a candidate structure for the containment of microorganisms}

As the plasma membranes of apoptotic cells remain intact, apoptotic cells contain intracellular $M t b$ and reduce their viability. One mechanism that sequesters $M t b$ within apoptotic cells and stabilizes the dying cell's plasma membrane is formation of the "apoptotic envelope," a high $\mathrm{M}_{\mathrm{r}}$, cross-linked polymeric cell-surface protein layer. ${ }^{40}$ This process, which is initiated following infection of macrophages by avirulent $M t b$ strains, commences with exposure of phosphatidylserine on the cell surface. Deposition of the phospholipid-binding protein annexin-1 on the cell-surface phosphatidylserine then follows. The cell surface-bound annexin-1 is cross-linked through its amino-terminal domain by transglutaminases, resulting in a high $\mathrm{M}_{\mathrm{r}}$ annexin-1 polymer mesh encasing the dying cell. In contrast, on macrophages infected with virulent $\mathrm{H} 37 \mathrm{Rv}$, the amino-terminal domain of annexin-1 is proteolytically cleaved before cross-linking, which impairs formation of the apoptotic envelope, and makes the cell susceptible to necrosis. This subversive strategy of inhibiting the formation of the apoptotic envelope, which has been observed so far only in infection of macrophages with $M t b$ constitutes an additional mechanism by which virulent $M t b$ prevents apoptosis and instead induces necrosis of infected macrophages.

\section{HOW IS PROGRAMMED MACROPHAGE DEATH REGULATED? \\ ROS-dependent apoptosis in Mtb infection}

ROS operate within cells as signaling molecules. ${ }^{41}$ The purified protein derivative from $M t b$ induces Toll-like receptor-2dependent ROS production. ${ }^{42}$ In $M t b$-infected macrophages, ROS induces proinflammatory responses through activation of the apoptosis signal-regulating kinase, which is a member of the MAPKKK (mitogen-activated protein kinase kinase kinase (MAP3K)) family that activates both the Jun N-terminal kinase and the p38 pathways. ${ }^{43,44}$ Phagosomal ROS, which is generated after $M t b$ infection by the action of NOX2, is required for increased TNF synthesis, which is indispensable for the induction of apoptosis and anti-mycobacterial defenses. ${ }^{45}$ Accordingly, pathogens have developed strategies to counteract NOX2 function and suppress generation of phagosomal ROS. The nuoG gene encodes the NuoG subunit of the type I NADH (nicotinamide adenine dinucleotide)-dehydrogenase of $M t b$. Deletion of this gene leads to elevated ROS levels and apoptosis following infection of primary macrophages. ${ }^{11}$ Similarly, deletion of the secA2 gene, which encodes a protein required for the secretion of superoxide dismutase A, results in enhanced ROS production, apoptosis, and loss of virulence. ${ }^{10}$ Superoxide 


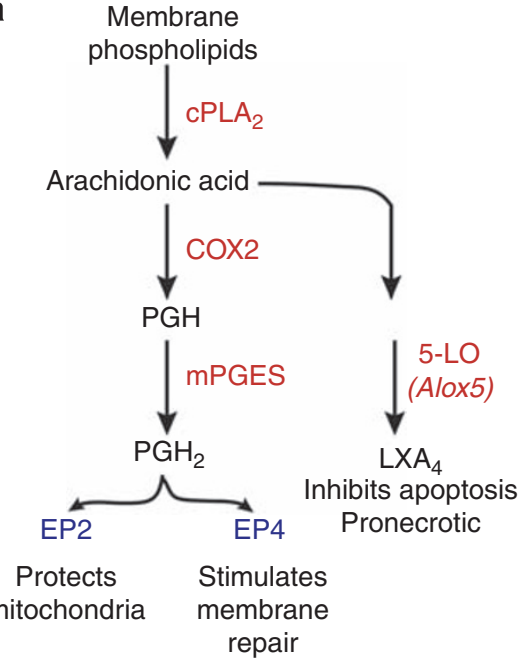

b

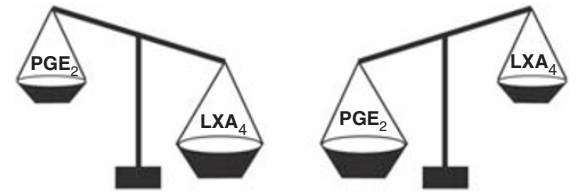

Less mitochondrion protection More membrane damage

Necrosis dominates
More mitochondrion protection More membrane repair Necrosis inhibited Apoptosis dominates

Figure 4 The role of eicosanoids in regulating death of infected macrophages. (a) Simplified version of eicosanoid biosynthesis. Substrates and products are indicated in black text. Enzymes are in red, and receptors in blue. (b) The balance of prostanoid and lipoxin production determines the death modality of $M t b$-infected macrophages. Excess production of $L X A_{4}$ leads to necrosis. In contrast, when $P G E_{2}$ production predominates, necrosis is inhibited and more apoptosis is the consequence. COX2, cyclooxygenase-2; CPLA $A_{2}$, cytosolic phospholipase $A_{2}$; 5-LO, 5-lipoxygenase;

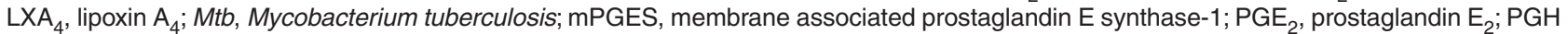
prostaglandin $\mathrm{H}$.

dismutase A breaks down superoxide leading to diminished ROS generation, which is a mechanism that $M t b$ uses to prevent apoptosis.

Although plasma membrane-associated ROS production might contribute to TNF-induced necrosis under certain circumstances, ${ }^{46}$ phagosomal ROS production is clearly associated with TNF-dependent apoptosis. ${ }^{11,45}$ In contrast, it was shown in the early 1990s that mitochondrial ROS production leads to a TNFdependent necrotic response. ${ }^{47}$ Recent data demonstrating that RIP-3 regulates downstream mitochondrial ROS generation during necrosis confirm these early findings. ${ }^{48}$ The manner by which ROS produced by the plasma membrane, phagolysosome, or mitochondrion, is differentially regulated and contributes to the death modality of the $M t b$-infected cell is not clearly understood.

\section{Role of eicosanoids in mitochondrial protection}

Arachidonic acid is the precursor of a group of common lipid mediators referred to as eicosanoids, which include prostanoids and LXs. Arachidonic acid, which is generated by the action of phospholipases on cell membrane phospholipids ${ }^{29}$ is involved in the induction of apoptosis in various cell types. ${ }^{49}$ The importance of this potential mechanism to induce cell death in vivo is not clear.

Recent work has focused on the role of the eicosanoid prostaglandin (PG) $\mathrm{E}_{2}$ and LXs in the regulation of programmed cell death in $M t b$-infected macrophages (Figure 4). PGs are synthesized by the action of the cyclooxygenases (COX), namely COX1 and COX2, which process arachidonic acid into PGH. ${ }^{50}$ Specific synthases act on $\mathrm{PGH}$ to produce various prostanoid species, which include $\mathrm{PGD}_{2}, \mathrm{PGE}_{2}, \mathrm{PGF}_{2} \alpha, \mathrm{PGI}_{2}$, and thromboxane. $\mathrm{PGE}_{2}$ is a well-known PG, the biological activity of which is largely defined by interaction with its four specific receptors $\mathrm{EP} 1, \mathrm{EP} 2, \mathrm{EP} 3$, and EP4. Of special interest here is EP2, which is involved in inflammatory responses, and EP4, which promotes Th1 differentiation and Th17 expansion. ${ }^{51}$ Both receptors upregulate intracellular cyclic adenosine monophosphate levels and are involved in inflammatory responses, ${ }^{52}$ but EP2 signaling is mediated by protein kinase $\mathrm{A},{ }^{53}$ whereas EP4 signals primarily through a phosphoinositide 3-kinase-dependent pathway. ${ }^{54}$

$\mathrm{PGE}_{2}$ production is known to occur in lipid bodies, which are formed intracellularly after infection of murine macrophages with BCG..$^{55}$ Other avirulent $M t b$ strains such as H37Ra induce $\mathrm{PGE}_{2}$ production, which protects the infected macrophage against necrosis. When $\mathrm{PGE}_{2}$ engages the macrophage EP2 receptor, it initiates protection of the mitochondria from bacteria-induced damage. Add-back experiments have shown that $\mathrm{PGE}_{2}$ prevents the macrophage mitochondria from damage in $\mathrm{PGES}^{-1-}$ murine macrophage infected with $M t b$, indicating that $\mathrm{PGE}_{2}$ is the active protective component. ${ }^{4}$ In contrast, engagement of EP4 stimulates plasma membrane repair mediated by the recruitment of lysosomal membranes to the cell surface of infected macrophages (see below). ${ }^{7}$ Plasma membrane repair is inhibited by virulent $M t b$ infection, which is another mechanism that contributes to the necrotic fate of infected macrophages.

Although H37Ra stimulates $\mathrm{PGE}_{2}$, virulent $M t b$ induces the production of $\mathrm{LXA}_{4}$ by macrophages. ${ }^{8} \mathrm{LXA}_{4}$ is an important factor involved in the resolution of inflammation ${ }^{56}$ and stimulates phagocytosis of apoptotic neutrophils by macrophages. ${ }^{57}$ By inhibiting COX2 expression and the generation of $\mathrm{PGE}_{2}$, $\mathrm{LXA}_{4}$ renders the infected macrophage more prone to necrosis. ${ }^{56}$ Pronecrotic functions of $\mathrm{LXA}_{4}$ also include inhibition of apoptosis induced by lipopolysaccharide/interferon- $\gamma$, inhibition of the release of proapoptotic mediators from the mitochondria, and inhibition of caspase activation and synthesis of ROS. ${ }^{58}$ These additional data establish $\mathrm{LXA}_{4}$ as an important mediator of necrosis. 


\section{$\mathrm{PGE}_{2}$ as a regulator of cell death}

Our studies identify the fate of the macrophage following $M t b$ infection as an essential component of the immune response against the pathogen. To our knowledge, this is the first example of pathways involved in the resolution of inflammation that are subverted by a pathogen to thwart innate immune defenses. It is noteworthy that diverse cellular pathways that are dependent on the function of $\mathrm{PGE}_{2}$ all have in common that they promote apoptosis and prevent necrosis of the host macrophage after $M t b$ infection. These pathways include the synthesis of COX2, the inducible PGH synthase involved in $\mathrm{PGE}_{2}$ production, the synthesis of plasminogen activator inhibitor type 2 (serpin $\mathrm{B} 2$ ), a serpin protease inhibitor that blocks truncation of annexin-1 on the macrophage surface protecting against necrosis, ${ }^{40}$ induction of synaptotagmin-7 (Syt-7) transcription, the essential lysosomal $\mathrm{Ca}^{++}$sensor required for fusion of the plasma membrane with lysosomal vesicles necessary for induction of apoptosis, ${ }^{7}$ and protection of the mitochondrial inner membrane from irreversible damage by MPT caused by virulent $M t b$ strains. ${ }^{8}$ Thus, a diverse set of mechanisms all leading to apoptosis are dependent on $\mathrm{PGE}_{2}$, and the balance between $\mathrm{PGE}_{2}$ and $\mathrm{LXA}_{4}$ determines the relative proportion of cells undergoing apoptosis or necrosis (Figure $4 \mathbf{b}$ ).

\section{Virulent $\boldsymbol{M}$ tb inhibits plasma membrane repair}

Early secreted antigen 6 kilodaltons (ESAT-6) secretion system-1 is a specialized type VII secretion system that is required by $M t b$ for the secretion of certain virulence factors, including the immunodominant antigens ESAT6 and CFP10. Although known to contribute to bacterial virulence, why ESAT- 6 secretion system-1 is required for bacterial survival in the host is unknown. Some data indicate that ESAT6 damages host cell membranes. ${ }^{59,60} \mathrm{We}$ hypothesized that disruption of the plasma membrane by $M t b$ is one mechanism that induces necrosis of the macrophage. Membrane lesions are rapidly repaired by a highly conserved mechanism that depends on exocytosis of endomembranes derived from lysosomes and Golgi apparatus. ${ }^{7}$ As exocytosis of lysosomes depends on the function of the calcium sensor Syt-7, we considered whether Syt-7 is required for exocytosis of lysosomes and repair of plasma membrane lesions in macrophages infected with $M t b$.

Indeed, plasma membrane microdisruptions generated by the avirulent strain $\mathrm{H} 37 \mathrm{Ra}$ are rapidly resealed by recruitment of lysosomes and Golgi-derived vesicles to the plasma membrane of the infected macrophage. ${ }^{7}$ Whereas Syt-7 is the principle calcium sensor of the lysosome, exocytosis of Golgi-derived vesicles is dependent on the calcium sensor NCS-1 (neuronal calcium sensor-1) and can be blocked by brefeldin A. Exocytosis of both lysosomal and Golgi-derived vesicles is independently required for the induction of apoptosis as either block of lysosome recruitment by Syt-7 gene silencing or block of Golgi-vesicle recruitment by brefeldin A causes necrosis of infected cells. ${ }^{8}$ Importantly, plasma membrane repair by lysosomal exocytosis is dependent on the production of $\mathrm{PGE}_{2}$, which induces Syt-7 transcription. In contrast to infection with attenuated H37Ra, virulent H37Rv downregulates lysosomal and Golgi-vesicledependent plasma membrane repair. Thus, inhibition of plasma membrane repair by virulent $M t b$ is based on its capacity to block PGE $_{2}$ synthesis and constitutes an efficient mechanism to induce necrosis, and to enable the bacilli to spread into new cells.

\section{CONCLUSIONS}

The finding that macrophages infected with virulent $M t b$ undergo necrosis, whereas those infected with attenuated mutant strains of $M t b$ undergo apoptosis, suggests that wildtype $M t b$ actively inhibits apoptosis. This forms the foundation for the concept that apoptosis is an innate macrophage defense mechanism, a concept supported by the finding that apoptosis reduces the viability of $M t b$ and provides an important link to the establishment of T-cell immunity. Investigation of the interaction between $M t b$ and macrophages finds that three distinct mechanisms contribute to macrophage necrosis. First, $M t b$ inhibits plasma membrane repair. Second, virulent $M t b$ causes inner mitochondrial membrane damage. Third, virulent $M t b$ inhibits cross-linking of annexin-1, which impairs formation of the apoptotic envelope. These three effects predispose the infected macrophage to necrosis. In part, these events occur because virulent $M t b$ inhibits the production of $\mathrm{PGE}_{2}$, a PG that is important for stimulation of membrane repair and protection of the mitochondrion. The manner by which virulent $M t b$ subverts eicosanoid biosynthesis to alter the death modality of macrophages to foil innate immunity is an important area for future investigation. Given our capacity to manipulate these pathways, a better understanding of how their regulation is altered by Mycobacteria may lead to novel ways to intervene therapeutically. Our capacity to develop immunomodulatory strategies based on these pathways to enhance vaccine efficacy may be just as promising.

\section{ACKNOWLEDGMENTS}

This work was supported by the National Institutes of Health grants Al50216 and Al072143 to HGR, and R01 Al47171 to SMB.

\section{DISCLOSURE}

The authors declared no conflict of interest.

C 2011 Society for Mucosal Immunology

\section{REFERENCES}

1. Kang, B.K. \& Schlesinger, L.S. Characterization of mannose receptordependent phagocytosis mediated by Mycobacterium tuberculosis lipoarabinomannan. Infect. Immun. 66, 2769-2777 (1998).

2. Schlesinger, L.S., Kaufman, T.M., lyer, S., Hull, S.R. \& Marchiando, L.K. Differences in mannose receptor-mediated uptake of lipoarabinomannan from virulent and attenuated strains of Mycobacterium tuberculosis by human macrophages. J. Immunol. 157, 4568-4575 (1996).

3. Sturgill-Koszycki, S. et al. Lack of acidification in Mycobacterium phagosomes produced by exclusion of the vesicular proton-ATPase [see comments] [published erratum appears in Science 199411 March;263(5152):1359]. Science 263, 678-681 (1994).

4. Chen, M., Gan, H. \& Remold, H.G. A mechanism of virulence: virulent Mtb tuberculosis strain H37Rv but not attenuated H37Ra causes significant mitochondrial inner membrane disruption in macrophages leading to necrosis. J. Immunol. 176, 3707-3716 (2006).

5. Molloy, A., Laochumroonvorapong, P. \& Kaplan, G. Apoptosis, but not necrosis, of infected monocytes is coupled with killing of intracellular bacillus Calmette-Guerin. J. Exp. Med. 180, 1499-1509 (1994). 
6. Fratazzi, C., Arbeit, R.D., Carini, C. \& Remold, H.G. Programmed cell death of Mycobacterium avium serovar 4-infected human macrophages prevents the mycobacteria from spreading and induces mycobacterial growth inhibition by freshly added, uninfected macrophages. J. Immunol. 158, 4320-4327 (1997).

7. Divangahi, M. et al. Mycobacterium tuberculosis evades macrophage defenses by inhibiting plasma membrane repair. Nat. Immunol. 10, 899906 (2009)

8. Chen, M. et al. Lipid mediators in innate immunity against tuberculosis: opposing roles of PGE2 and LXA4 in the induction of macrophage death. J. Exp. Med. 205, 2791-2801 (2008).

9. Oddo, M. et al. Fas ligand-induced apoptosis of infected human macrophages reduces the viability of intracellular Mycobacterium tuberculosis. J. Immunol. 160, 5448-5454 (1998).

10. Hinchey, J. et al. Enhanced priming of adaptive immunity by a proapoptotic mutant of Mycobacterium tuberculosis. J. Clin. Invest. 117, 2279-2288 (2007).

11. Velmurugan, K. et al. Mycobacterium tuberculosis nuoG is a virulence gene that inhibits apoptosis of infected host cells. PLoS Pathog. 3, e110 (2007).

12. Winau, F. et al. Apoptotic vesicles crossprime CD8 T cells and protect against tuberculosis. Immunity 24, 105-117 (2006).

13. Divangahi, M. et al. NOD2-deficient mice have impaired resistance to Mycobacterium tuberculosis infection through defective innate and adaptive immunity. J. Immunol. 181, 7157-7165 (2008).

14. Maertzdorf, J. et al. Human gene expression profiles of susceptibility and resistance in tuberculosis. Genes Immun. 12, 15-22 (2011).

15. Abebe, M. et al. Expression of apoptosis-related genes in an Ethiopian cohort study correlates with tuberculosis clinical status. Eur. J. Immunol. 40, 291-301 (2010).

16. Tobin, D.M. et al. The Ita4h locus modulates susceptibility to mycobacterial infection in zebrafish and humans. Cell 140, 717-730 (2010).

17. Herb, F. et al. ALOX5 variants associated with susceptibility to human pulmonary tuberculosis. Hum. Mol. Genet. 17, 1052-1060 (2008).

18. Cohen, J.J. Apoptosis. Immunol. Today 14, 126-130 (1993).

19. Fadok, V.A. et al. Exposure of phosphatidylserine on the surface of apoptotic lymphocytes triggers specific recognition and removal by macrophages. J. Immunol. 148, 2207-2216 (1992).

20. Coleman, M.L. et al. Membrane blebbing during apoptosis results from caspase-mediated activation of ROCK I. Nat. Cell Biol. 3, 339-345 (2001).

21. Henson, P.M. \& Tuder, R.M. Apoptosis in the lung: induction, clearance and detection. Am. J. Physiol. Lung Cell Mol. Physiol. 294, L601-L611 (2008).

22. Bergsbaken, T., Fink, S.L. \& Cookson, B.T. Pyroptosis: host cell death and inflammation. Nat. Rev. Microbiol. 7, 99-109 (2009).

23. Vandenabeele, P., Galluzzi, L., Vanden Berghe, T. \& Kroemer, G. Molecular mechanisms of necroptosis: an ordered cellular explosion. Nat. Rev. Mol. Cell Biol. 11, 700-714 (2010).

24. Christofferson, D.E. \& Yuan, J. Necroptosis as an alternative form of programmed cell death. Curr. Opin. Cell Biol. 22, 263-268 (2010).

25. Thornberry, N.A. \& Lazebnik, Y. Caspases: enemies within. Science $\mathbf{2 8 1}$ 1312-1316 (1998).

26. Duan, L., Gan, H., Arm, J. \& Remold, H.G. Cytosolic phospholipase A2 participates with TNF-alpha in the induction of apoptosis of human macrophages infected with Mycobacterium tuberculosis H37Ra. J. Immunol. 166, 7469-7476 (2001)

27. Hart, P.H. et al. Potential antiinflammatory effects of interleukin 4 : suppression of human monocyte tumor necrosis factor alpha, interleukin 1, and prostaglandin E2. Proc. Natl Acad. Sci. USA 86, 3803-3807 (1989).

28. Balcewicz-Sablinska, M.K., Keane, J., Kornfeld, H. \& Remold, H.G. Pathogenic mycobacterium tuberculosis evades apoptosis of host macrophages by release of TNF-R2, resulting in inactivation of TNF-\{alpha\}. J. Immunol. 161, 2636-2641 (1998).

29. Dennis, E.A. The growing phospholipase A2 superfamily of signal transduction enzymes. Trends Biochem. Sci. 22, 1-2 (1997)

30. Kundu, M. et al. A TNF- and c-Cbl-dependent FLIP(S)-degradation pathway and its function in Mycobacterium tuberculosis-induced macrophage apoptosis. Nat. Immunol. 10, 918-926 (2009).

31. Green, D.R. \& Kroemer, G. The pathophysiology of mitochondrial cell death. Science 305, 626-629 (2004).
32. Kim, J.S., He, L. \& Lemasters, J.J. Mitochondrial permeability transition: a common pathway to necrosis and apoptosis. Biochem. Biophys. Res Commun. 304, 463-470 (2003).

33. Chipuk, J.E. \& Green, D.R. How do BCL-2 proteins induce mitochondrial outer membrane permeabilization? Trends Cell Biol. 18, 157-164 (2008).

34. Bossy-Wetzel, E., Newmeyer, D.D. \& Green, D.R. Mitochondrial cytochrome $c$ release in apoptosis occurs upstream of DEVD-specific caspase activation and independently of mitochondrial transmembrane depolarization. EMBO J. 17, 37-49 (1998).

35. Ricci, J.E. et al. Disruption of mitochondrial function during apoptosis is mediated by caspase cleavage of the $\mathrm{p} 75$ subunit of complex I of the electron transport chain. Cell 117, 773-786 (2004).

36. Martinvalet, D., Dykxhoorn, D.M., Ferrini, R. \& Lieberman, J. Granzyme A cleaves a mitochondrial complex I protein to initiate caspase-independent cell death. Cell 133, 681-692 (2008).

37. Baines, C.P. et al. Loss of cyclophilin D reveals a critical role for mitochondrial permeability transition in cell death. Nature 434, 658-662 (2005).

38. Gan, H. et al. Enhancement of antimycobacterial activity of macrophages by stabilization of inner mitochondrial membrane potential. J. Infect. Dis. 191, 1292-1300 (2005).

39. Connern, C.P. \& Halestrap, A.P. Purification and N-terminal sequencing of peptidyl-prolyl cis-trans-isomerase from rat liver mitochondrial matrix reveals the existence of a distinct mitochondrial cyclophilin. Biochem. J. 284 (Part 2), 381-385 (1992).

40. Gan, H. et al. Mycobacterium tuberculosis blocks crosslinking of annexin1 and apoptotic envelope formation on infected macrophages to maintain virulence. Nat. Immunol. 9, 1189-1197 (2008).

41. Finkel, T. Oxidant signals and oxidative stress. Curr. Opin. Cell Biol. 15, 247-254 (2003).

42. Yang, C.S. et al. ASK1-p38 MAPK-p47phox activation is essential for inflammatory responses during tuberculosis via TLR2-ROS signalling. Cell Microbiol. 10, 741-754 (2008).

43. Ichijo, H. et al. Induction of apoptosis by ASK1, a mammalian MAPKKK that activates SAPK/JNK and p38 signaling pathways. Science $\mathbf{2 7 5}$ 90-94 (1997).

44. Takeda, K., Noguchi, T., Naguro, I. \& Ichijo, H. Apoptosis signal-regulating kinase 1 in stress and immune response. Annu. Rev. Pharmacol. Toxicol. 48, 199-225 (2008).

45. Miller, J.L., Velmurugan, K., Cowan, M.J. \& Briken, V. The type I NADH dehydrogenase of Mycobacterium tuberculosis counters phagosomal NOX2 activity to inhibit TNF-alpha-mediated host cell apoptosis. PLoS Pathog. 6, e1000864 (2010).

46. Kim, Y.S., Morgan, M.J., Choksi, S. \& Liu, Z.G. TNF-induced activation of the Nox1 NADPH oxidase and its role in the induction of necrotic cell death. Mol. Cell 26, 675-687 (2007)

47. Schulze-Osthoff, K. et al. Cytotoxic activity of tumor necrosis factor is mediated by early damage of mitochondrial functions. Evidence for the involvement of mitochondrial radical generation. J. Biol. Chem. 267, 5317-5323 (1992)

48. Cho, Y.S. et al. Phosphorylation-driven assembly of the RIP1-RIP3 complex regulates programmed necrosis and virus-induced inflammation. Cell 137, 1112-1123 (2009).

49. Wolf, L.A. \& Laster, S.M. Characterization of arachidonic acid-induced apoptosis. Cell Biochem. Biophys. 30, 353-368 (1999).

50. Rocca, B. \& FitzGerald, G.A. Cyclooxygenases and prostaglandins: shaping up the immune response. Int. Immunopharmacol. 2, 603-630 (2002).

51. Yao, C. et al. Prostaglandin E2-EP4 signaling promotes immune inflammation through Th1 cell differentiation and Th17 cell expansion. Nat. Med. 15, 633-640 (2009).

52. Honda, T., Segi-Nishida, E., Miyachi, Y. \& Narumiya, S. Prostacyclin-IP signaling and prostaglandin E2-EP2/EP4 signaling both mediate joint inflammation in mouse collagen-induced arthritis. J. Exp. Med. 203, 325-335 (2006).

53. Fujino, H., West, K.A. \& Regan, J.W. Phosphorylation of glycogen synthase kinase-3 and stimulation of T-cell factor signaling following activation of EP2 and EP4 prostanoid receptors by prostaglandin E2 J. Biol. Chem. 277, 2614-2619 (2002).

54. Fujino, H., Xu, W. \& Regan, J.W. Prostaglandin E2 induced functional expression of early growth response factor- 1 by EP4, but not EP2, prostanoid receptors via the phosphatidylinositol 3-kinase and extracellular signal-regulated kinases. J. Biol. Chem. 278, 12151-12156 (2003). 
55. D'Avila, H. et al. Mycobacterium bovis bacillus Calmette-Guerin induces TLR2-mediated formation of lipid bodies: intracellular domains for eicosanoid synthesis in vivo. J. Immunol. 176, 3087-3097 (2006).

56. Levy, B.D., Clish, C.B., Schmidt, B., Gronert, K. \& Serhan, C.N. Lipid mediator class switching during acute inflammation: signals in resolution. Nat. Immunol. 2, 612-619 (2001).

57. Godson, C. et al. Cutting edge: lipoxins rapidly stimulate nonphlogistic phagocytosis of apoptotic neutrophils by monocyte-derived macrophages. J. Immunol. 164, 1663-1667 (2000).
58. Prieto, P. et al. Lipoxin A4 impairment of apoptotic signaling in macrophages: implication of the PI3K/Akt and the ERK/Nrf-2 defense pathways. Cell Death Differ. 17, 1179-1188 (2010).

59. Smith, J. et al. Evidence for pore formation in host cell membranes by ESX-1-secreted ESAT- 6 and its role in Mycobacterium marinum escape from the vacuole. Infect. Immun. 76, 5478-5487 (2008).

60. de Jonge, M.I. et al. ESAT-6 from Mycobacterium tuberculosis dissociates from its putative chaperone CFP-10 under acidic conditions and exhibits membrane-lysing activity. J. Bacteriol. 189, 6028-6034 (2007). 